\title{
Tumor response assessment by the single-lesion measurement per organ in small cell lung cancer
}

\author{
Soong Goo Jung ${ }^{1}$, Jung Han Kim ${ }^{1}$, Hyeong Su Kim ${ }^{1}$, Kyoung Ju Kim², Ik Yang ${ }^{3}$ \\ ${ }^{1}$ Department of Internal Medicine, ${ }^{2}$ Department of Radiation Oncology, ${ }^{3}$ Department of Radiology, Kangnam Sacred-Heart Hospital, Hallym \\ University Medical Center, Hallym University College of Medicine, Seoul, Republic of Korea \\ Correspondence to: Jung Han Kim, MD, PhD. Department of Internal Medicine, Kangnam Sacred-Heart Hospital, Hallym University Medical \\ Center, Shingil-ro 1, Youngdeungpo-Gu, Seoul, 07441, Republic of Korea. Email: harricil@hotmail.com or harricil@hallym.or.kr.
}

\begin{abstract}
Background: The criterion of two target lesions per organ in the Response Evaluation Criteria in Solid Tumors (RECIST) version 1.1 is an arbitrary one, being supported by no objective evidence. The optimal number of target lesions per organ still needs to be investigated. We compared tumor responses using the RECIST 1.1 (measuring two target lesions per organ) and modified RECIST 1.1 (measuring the single largest lesion in each organ) in patients with small cell lung cancer (SCLC).

Methods: We reviewed medical records of patients with SCLC who received first-line treatment between January 2004 and December 2014 and compared tumor responses according to the two criteria using computed tomography.

Results: There were a total of 34 patients who had at least two target lesions in any organ according to the RECIST 1.1 during the study period. The differences in the percentage changes of the sum of tumor measurements between RECIST 1.1 and modified RECIST 1.1 were all within $13 \%$. Seven patients showed complete response and fourteen showed partial response according to the RECIST 1.1. The overall response rate was $61.8 \%$. When assessing with the modified RECIST 1.1 instead of the RECIST 1.1, tumor responses showed perfect concordance between the two criteria $(\mathrm{k}=1.0)$.

Conclusions: The modified RECIST 1.1 showed perfect agreement with the original RECIST 1.1 in the assessment of tumor response of SCLC. Our result suggests that it may be enough to measure the single largest target lesion per organ for evaluating tumor response.
\end{abstract}

Keywords: Target lesion; Response Evaluation Criteria in Solid Tumors 1.1 (RECIST 1.1); modified Response Evaluation Criteria in Solid Tumors; tumor response 1.1 (modified RECIST 1.1); small cell lung cancer (SCLC)

Submitted Aug 07, 2015. Accepted for publication Feb 19, 2016.

doi: 10.21147 /j.issn.1000-9604.2016.02.03

View this article at: http://dx.doi.org/10.21147/j.issn.1000-9604.2016.02.03

\section{Introduction}

As decision on the subsequent cancer treatments usually depends on radiologic changes in the tumor burden, the accurate assessment of tumor response is essential for patients receiving anti-cancer treatments. Since the early 1980s, the World Health Organization (WHO) response criteria were adopted as the standard method for evaluating tumor response (1). Tumor burden was assessed by the products of two-dimensional measurements. Baseline measurements were then compared with the follow-up measurements to determine tumor response. Because the details for selecting target lesions were not clearly described in the WHO guidelines, however, the assessment of tumor response was often poorly reproducible between investigators $(2,3)$.

In 2000, the Response Evaluation Criteria in Solid Tumors (RECIST) Working Group proposed the RECIST guideline version 1.0 (RECIST 1.0) as a new set of tumor response criteria (4). The original RECIST 1.0 clearly defined the minimum size of target lesion by computed tomography (CT) and incorporated uni-dimensional 
measurement instead of the bi-dimensional method of WHO criteria for measuring tumor size. The RECIST 1.0 criteria adopted a total of ten target lesions with a maximum of five lesions per organ. However, a number of issues and questions on the RECIST 1.0 including the number of target lesions, the size of lymph nodes (LNs) to be measured, and the application of new imaging technologies such as multi-detector computed tomography (MDCT) and positron emission tomography (PET) has been raised (5).

Based on the analyses of the database of about 6,500 patients with more than 18,000 target lesions (6), the RECIST Working Group published a revised version of the RECIST guidelines (RECIST 1.1) in January 2009 (7). The important changes included the maximum number of target lesions, the LN measurements, and the definition of disease progression $(8,9)$. The maximum number of target lesions to be assessed has been reduced from ten to five in total, with a maximum of two target lesions per organ instead of five. While the total of ten target lesions in the RECIST 1.0 was arbitrarily selected, the RECIST 1.1 defined a total of five lesions through the patients' data analysis (6) and statistical simulating studies $(10,11)$. However, the criterion of two target lesions per organ was still an arbitrary decision. Therefore, the optimal number of target lesions per organ need to be investigated in further studies.

Under the condition of accurately assessing the changes of tumor burden, it is desirable to simplify the guidelines for assessing tumor response as far as possible. Before the RECIST 1.1 was presented, Zacharia and colleagues had reported that measuring the single largest lesion of hepatic metastases yielded almost the same response classification as measuring up to five hepatic lesions in patients with colorectal cancer (CRC) (12). Based on this finding, we assumed that measuring the single largest lesion in each organ (modified RECIST 1.1; mRECIST 1.1) might show almost the same response classification as measuring two target lesions per organ (RECIST 1.1). Recently we compared the tumor responses between the RECIST 1.1 and mRECIST 1.1 in patients with advanced or metastatic non-small cell lung cancer (NSCLC) (13), gastric cancer (GC) (14), and CRC (15), and found that the mRECIST 1.1 , with a decreased total number of target lesions to be measured, was comparable to the original RECIST 1.1 in the assessment of tumor responses in patients with metastatic tumors (13-16).

Lung cancer is one of the most common forms of cancer in the world, in terms of incidence and mortality.
The previous study was conducted in patients only with advanced NSCLC (13). Small cell lung cancer (SCLC) accounts for approximately $15 \%$ of all primary malignancies occurring in the thorax $(17,18)$. In this study, we compared the tumor responses by CT between the RECIST 1.1 and mRECIST 1.1 in patients with SCLC. Our aim was to test whether the single-lesion measurement per organ yields the same response classification.

\section{Patients and methods}

\section{Patients}

This study was approved by the Institutional Review Board with a waiver of patients' informed consent. We reviewed the medical records of patients with SCLC who received chemoradiation or first-line chemotherapy between January 1, 2004 and December 31, 2014 at Kangnam SacredHeart Hospital, Seoul, South Korea. The patient was eligible for the study if he or she had the following criteria; histologically confirmed small cell carcinoma of the lung, having at least two measurable lesions in any organ by the RECIST version 1.1, no history of other cancer, no history of previous chemotherapy or radiotherapy, and CT tumor assessments at baseline and after chemoradiation or chemotherapy. The diagnosis of SCLC was based on the histologic appearance of round to spindle-shaped small cells with dense nuclei, inconspicuous nucleoli, and sparse cytoplasm. For equivocal cases by the histologic criteria, immunohistochemistries by avidin-biotin complex method were performed to support the accurate diagnosis using antibodies to chromogranin A, synaptophysin, or neuronespecific enolase (NSE) (DAKO cytomation, Glostrup, Denmark). Patients with well differentiated neuroendocrine tumor or mixed histologic types were excluded. Patients who had shown the progression of non-target lesions or developed new lesions at the follow-up CT were also excluded from the final analysis.

During the study period, a total of 42 patients with SCLC received the first-line treatment with chemoradiation or chemotherapy. Five patients (11.9\%) had only one target lesion per organ according to the RECIST 1.1, and one discontinued treatment before tumor response evaluation. According to the inclusion criteria, two patients (4.8\%) who showed the progression of non-target lesion or development of new lesions were also excluded from the study. Finally, a total of 34 patients $(80.9 \%)$ who had at least two measurable lesions in any one organ were included in the analyses. 


\section{CT examinations}

All CT scans were obtained on the MDCT scanner (SOMATOM Sensation, Giemens Healthcare, Germany) with injection of $80 \mathrm{~mL}$ (at a rate of $3 \mathrm{~mL} / \mathrm{s}$ ) of contrast medium, iopromide (Ultravist 300, Bayer Bayer Schering, Germany), with a scanning delay of 25-30 s. The CT images were reconstructed with a slice thickness of $5 \mathrm{~mm}$ and were uploaded on the Picture Archiving and Communication System (PACS) workstation (PiView Star, INFINITT Healthcare Co. LTD., Seoul, South Korea).

\section{Tumor measurements}

The CT scans for assessing tumor response were obtained at base and about 2 months after first-line anti-cancer treatment, and tumor responses were determined with no interval confirmation. Two investigators re-evaluated each patient's tumor measurements from the original CT images using the RECIST 1.1 and mRECIST 1.1, respectively. Tumors were measured manually on axial CT image planes using calipers of the measurement tool on the PACS. LN measurement was performed in its short axis, considering $\mathrm{LN}$ of at least $15 \mathrm{~mm}$ to be a target lesion. $\mathrm{LN}$ with at least $10 \mathrm{~mm}$ but less than $15 \mathrm{~mm}$ in its short axis was considered as a non-target lesion, and $\mathrm{LN}$ with a short axis of less than $10 \mathrm{~mm}$ was regarded as normal. The maximum number of target lesions to be assessed was five in total, with a maximum of two per organ (RECIST 1.1) or a single largest lesion in each organ (mRECIST 1.1). The target lesion description and size measurement, the sum of the longest diameters of the target lesions, the development of new lesions, the description of non-target lesions, and the tumor response for each patient were recorded by consensus of the two investigators. For cases showing a significant discrepancy between the two investigators, a board-certified chest radiologist re-evaluated the CT images. The definitions of tumor response were in accordance with the original RECIST version 1.1 (7).

\section{Statistical analysis}

A paired student's $t$-test was used to estimate the statistical significance of changes in the number of target lesions between the RECIST 1.1 and mRECIST 1.1. All P values were based on a two-sided hypothesis, with a value of less than 0.05 being considered significant. The level of concordance in the tumor responses between the two criteria
Table 1 Characteristics of the 34 patients

\begin{tabular}{lc}
\hline Characteristics & No. of patients (\%) \\
\hline Median age, years [range] & $69[54-79]$ \\
Gender & $29(85.3)$ \\
Male & $5(14.7)$ \\
Female & \\
Stage & $10(29.4)$ \\
Limited & $24(70.6)$ \\
Extensive & \\
Measurable target lesions & $25(73.5)$ \\
Lungs & $32(94.1)$ \\
Lymph nodes & $9(26.5)$ \\
Liver & $3(8.8)$ \\
Adrenal gland & \\
First-line treatment & $18(52.9)$ \\
Etoposide + platinum & $6(17.6)$ \\
Irinotecan + platinum & $10(29.4)$ \\
Chemoradiation &
\end{tabular}

was assessed using kappa statistics. A kappa value of more than 0.75 was interpreted as showing strong concordance.

\section{Results}

\section{Patient characteristics}

Patients' baseline characteristics are presented in Table 1. The patients consisted of 29 males (85.3\%) and 5 females (14.7\%), with a median age of 69 years. Twenty-four (70.6\%) patients had extensive disease (ED) and the remaining ten showed limited disease (LD). Most patients had measurable lesions in the LNs (94.1\%) or lung $(73.5 \%)$. Nine patients $(26.5 \%)$ also had target lesions in the liver. Seventeen patients $(50 \%)$ had target lesions in two organs, most commonly in the lungs and LNs. Eight patients (23.5\%) had target lesions in three organs (lung, LNs, and liver or adrenal gland).

Ten patients with LD (29.4\%) received chemoradiation with etoposide plus platinum (cisplatin or carboplatin) as first-line treatment. Twenty-four patients with ED were treated with platinum plus etoposide or irinotecan.

\section{Number of target lesions}

The number of target lesions according to the mRECIST 


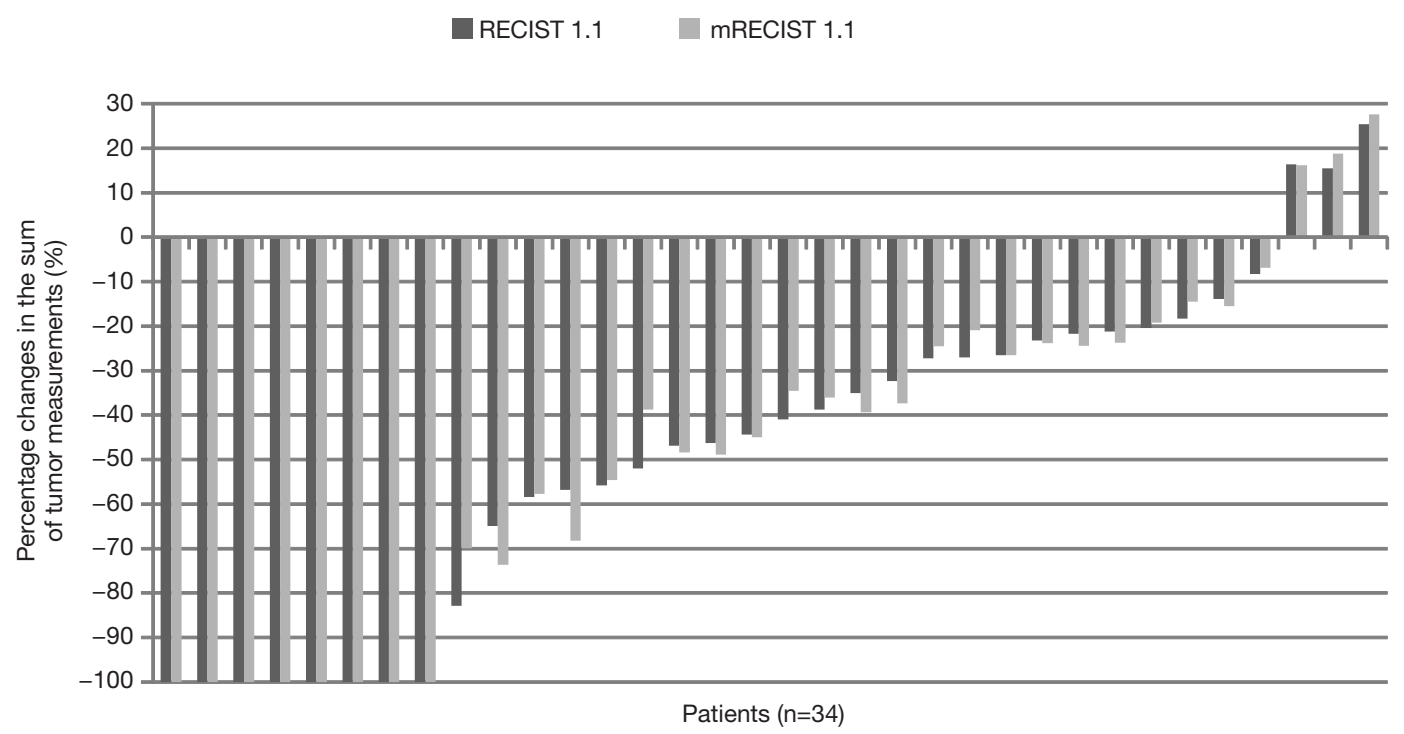

Figure 1 Percentage changes in the sum of tumor measurements according to the RECIST 1.1 versus modified RECIST 1.1 (mRECIST 1.1.).

Table 2 Tumor response assessment by the RECIST 1.1 versus modified RECIST1.1 (mRECIST1.1)

\begin{tabular}{lccccc}
\hline Tumor response & \multicolumn{4}{c}{ Tumor response by mRECIST 1.1 } & Total \\
\cline { 2 - 5 } by RECIST 1.1 & CR & PR & SD & 0 & 7 \\
\hline CR & 7 & 0 & 0 & 0 & 14 \\
PR & 0 & 14 & 0 & 0 & 12 \\
SD & 0 & 0 & 12 & 1 & 1 \\
PD & 0 & 0 & 0 & 1 & 34 \\
Total & 7 & 14 & 12 & 1 \\
\hline
\end{tabular}

The tumor responses show perfect agreement between the RECIST 1.1 and mRECIST 1.1. RECIST, Response Evaluation Criteria in Solid Tumors.

1.1 was significantly lower than that recorded according to the RECIST $1.1(\mathrm{P}<0.01)$. The median number of target lesions was 3 (range, 2-5) by the RECIST 1.1 and 2 (range, $1-4)$ by the mRECIST 1.1 , respectively. No patient showed metastatic sites with a newly defined target lesion by adopting the mRECIST 1.1, instead of the RECIST 1.1.

\section{Tumor responses}

The changes in the sum of tumor measurements respectively according to the RECIST 1.1 and mRECIST 1.1 are presented as percentages in Figure 1. The differences in the percentage changes of the sum of tumor measurements between the RECIST 1.1 and mRECIST 1.1 were all within $13 \%$ (range, $0-13 \%$ ).
The comparison of the tumor responses between the two criteria is shown in Table 2. Seven patients showed CR and 14 showed PR according to the RECIST 1.1. The overall response rate was $61.8 \%$. When assessing with the mRECIST 1.1 instead of the RECIST 1.1, the tumor responses showed perfect concordance between the two criteria $(\mathrm{k}=1.0)$.

\section{Discussion}

We investigated the impact of the single-lesion measurement per each organ (mRECIST 1.1), instead of two target lesions per organ (RECIST 1.1), on the tumor response in patients with SCLC. Although the mRECIST 1.1 significantly reduced the number of target lesions to 
be assessed, it has shown perfect concordance with the RECIST 1.1 in the assessment of tumor response.

Since both the WHO and RECIST guidelines mainly depend on the changes of tumor size on imaging studies in the assessment of tumor response, target lesions are the most important radiologic markers. The WHO criteria recommended measuring all target lesions with two dimensions (1). It would be ideal for assessing tumor response if all target lesions could be measured, but this is laborious in clinical practice. Therefore, it is reasonable to choose and follow the appropriate number of target lesions that can accurately reflect the changes of the overall tumor burden. The RECIST guideline 1.0 adopted a total of ten target lesions with a maximum of five per organ to be assessed (4). However, the maximum numbers of target lesions to be assessed were arbitrarily decided, with no objective evidence. The RECIST Working Group had analyzed the impact of assessing one, two, three or five target lesions, instead of ten, on the tumor response classification using their patient database (6). The results showed that assessing three or five target lesions led to no change in the overall response rate and progression-free survival, compared with measuring ten lesions according to the RECIST 1.0. Furthermore, statistical simulation studies also revealed little difference in the assessment of tumor response between five and ten target lesions $(10,11)$. Based on these results, the RECIST 1.1 adopted a total of five target lesions to be measured (7).

Although the total number of target lesions to be assessed was determined on the statistical simulation studies in the RECIST $1.1(10,11)$, the criterion of two lesions per organ was still an arbitrary value. We hypothesized that measuring the single largest lesion in each organ (mRECIST 1.1) might show almost the same response classification as measuring two target lesions per organ (RECIST 1.1). In the study of 64 patients with advanced NSCLC (13), three (4.7\%) showed disagreement in the assessment of tumor response according to the two criteria ( $\mathrm{k}=0.899)$ : two showed discordance between PR and SD and one between $\mathrm{PD}$ and SD. A pooled analysis (16) using the data of 153 patients from the three individual studies (13-15) indicated that the assessment of tumor response showed high concordance between the RECIST 1.1 and mRECIST 1.1 ( $\mathrm{k}=0.908)$, with only eight patients (5.2\%) showing disagreement between the two criteria.

In the current study, we compared tumor response assessment between the RECIST 1.1 and mRECIST 1.1 in patients with SCLC. Patients received chemotherapy or chemoradiation according to their disease status (LD or ED) in practical setting and tumor responses were assessed with no interval confirmation. As anticipated, the number of target lesions according to the mRECIST 1.1 was significantly lower than that according to the RECIST 1.1. Although this study had an important limitation with a small number of patients, the tumor responses showed perfect concordance between the two criteria $(\mathrm{k}=1.0)$. As we mentioned above, $5.2 \%$ of patients in the pool study showed the different response classification between the RECIST 1.1 and mRECIST 1.1 (16). In the current study with SCLC, however, no patients showed disagreement between the two criteria. More than $60 \%$ of patients with ED SCLC have shown tumor response (PR or CR) to first-line chemotherapy with platinum plus etoposide or irinotecan (19). When estimated regardless of disease stage (LD or ED) in our patients, the overall response rate was $61.8 \%$. This high chemosensitivity of SCLC may explain in part the perfect agreement of tumor responses between the two criteria in the current study. Our finding in SCLC, in concordance with the results of the previous studies with other type of cancers (12-15), suggests that it may be possible to measure the single largest target lesion per organ for assessing tumor response. On the assumption that both criteria are comparable in the assessment of tumor response, the mRECIST 1.1, with a decreased total number of target lesions, is expected to not only increase convenience but also decrease intra- and inter-observer variability in the measurement of target lesions.

Although the single-lesion measurement per organ has some advantages in the assessment of tumor response, it also has a couple of issues that need to be mentioned here. In clinical practice, although it is very rare, patients can show mixed tumor responses in which some tumors grow whereas others shrink (20-22). The mixed responses may lead to the discordance between the two criteria. There is also a concern that the largest target lesion may not always be the best one. Large lesions may be partially necrotic or contain cavitations and may not shrink to the same extent as smaller lesions (23). In addition, a number of target agents induce necrosis and cystic change in solid tumors without necessarily producing tumor shrinkage. In those cases, however, the RECIST 1.1 which measure two largest lesions per organ also has a potential risk that cannot exactly assess the changes of tumor burden. The RECIST 1.1 includes PET scans for the detection of new lesions. 18F-FDG PET is also increasingly adopted to monitor tumor responses to targeted therapies in solid tumor (24). It has been correlated with anatomical 
response and survival in patients treated with targeted agents for solid tumors $(25,26)$.

In conclusion, the modified RECIST 1.1 showed a perfect agreement with the original RECIST 1.1 in the assessment of tumor response of SCLC. Our results suggest that it may be enough to measure the single largest target lesion per organ for evaluating tumor response in clinical practice. Considering this study had a small number of patients with SCLC, however, our finding needs to be tested in larger studies.

\section{Acknowledgements}

None.

\section{Footnote}

Conflicts of Interest: The authors have no conflicts of interest to declare.

\section{References}

1. Miller AB, Hoogstraten B, Staquet M, et al. Reporting results of cancer treatment. Cancer 1981;47:207-14.

2. Therasse P. Measuring the clinical response. What does it mean? Eur J Cancer 2002;38:1817-23.

3. Choi JH, Ahn MJ, Rhim HC, et al. Comparison of WHO and RECIST criteria for response in metastatic colorectal carcinoma. Cancer Res Treat 2005;37:290-3.

4. Therasse P, Arbuck SG, Eisenhauer EA, et al. New guidelines to evaluate the response to treatment in solid tumors. European Organization for Research and Treatment of Cancer, National Cancer Institute of the United States, National Cancer Institute of Canada. J Natl Cancer Inst 2000;92:205-16.

5. Sargent DJ, Rubinstein L, Schwartz L, et al. Validation of novel imaging methodologies for use as cancer clinical trial end-points. Eur J Cancer 2009;45:290-9.

6. Bogaerts J, Ford R, Sargent D, et al. Individual patient data analysis to assess modifications to the RECIST criteria. Eur J Cancer 2009;45:248-60.

7. Eisenhauer EA, Therasse P, Bogaerts J, et al. New response evaluation criteria in solid tumours: revised RECIST guideline (version 1.1). Eur J Cancer 2009;45:228-47.

8. Schwartz LH, Bogaerts J, Ford R, et al. Evaluation of lymph nodes with RECIST 1.1. Eur J Cancer 2009;45:261-7.
9. Dancey JE, Dodd LE, Ford R, et al. Recommendations for the assessment of progression in randomised cancer treatment trials. Eur J Cancer 2009;45:281-9.

10. Moskowitz CS, Jia X, Schwartz LH, et al. A simulation study to evaluate the impact of the number of lesions measured on response assessment. Eur J Cancer 2009;45:300-10.

11. Schwartz LH, Mazumdar M, Brown W, et al. Variability in response assessment in solid tumors: effect of number of lesions chosen for measurement. Clin Cancer Res 2003;9:4318-23.

12. Zacharia TT, Saini S, Halpern EF, et al. CT of colon cancer metastases to the liver using modified RECIST criteria: determining the ideal number of target lesions to measure. AJR Am J Roentgenol 2006;186:1067-70.

13. Kim HS, Kim JH, Yang I. Tumor response assessment by measuring the single largest lesion per organ in patients with advanced non-small cell lung cancer. Lung Cancer 2014;85:385-9.

14. Kim HS, Kim JW, Kim JH, et al. Single-lesion measurement per organ for assessing tumor response in advanced gastric cancer. Oncology 2015;88:69-75.

15. Kim HS, Kim JH. Ideal number of target lesions per organ to measure in metastatic colorectal cancer. Oncol Lett 2014;8:1896-1900.

16. Jang HJ, Cho JW, Park B, et al. The assessment of tumor response by measuring the single largest lesion per organ in metastatic tumors: a pooled analysis of previously reported data. J Cancer 2015;6:169-76.

17. Houston KA, Henley SJ, Li J, et al. Patterns in lung cancer incidence rates and trends by histologic type in the United States, 2004-2009. Lung Cancer 2014;86:22-8.

18. Jung KW, Won YJ, Kong HJ, et al. Prediction of cancer incidence and mortality in Korea, 2014. Cancer Res Treat 2014;46:124-30.

19. Jiang J, Liang $X$, Zhou $X$, et al. A meta-analysis of randomized controlled trials comparing irinotecan/ platinum with etoposide/platinum in patients with previously untreated extensive-stage small cell lung cancer. J Thorac Oncol 2010;5:867-73.

20. Ryoo BY, Na II, Yang SH, et al. Synchronous multiple primary lung cancers with different response to gefitinib. Lung Cancer 2006;53:245-8.

21. Park KY, Jung JW, Nam SB, et al. Two lung masses with different responses to pemetrexed. Korean J Intern Med 2010;25:213-6.

22. Chen ZY, Zhong WZ, Zhang XC, et al. EGFR mutation heterogeneity and the mixed response to EGFR tyrosine 
kinase inhibitors of lung adenocarcinomas. Oncologist 2012;17:978-85.

23. Nishino M, Jagannathan JP, Ramaiya NH, et al. Revised RECIST guideline version 1.1: What oncologists want to know and what radiologists need to know. AJR Am J Roentgenol 2010;195:281-9.

24. Krystal GW, Alesi E, Tatum JL. Early FDG/PET scanning as a pharmacodynamic marker of anti-EGFR antibody activity in colorectal cancer. Mol Cancer Ther 2012;11:1385-8.

Cite this article as: Jung SG, Kim JH, Kim HS, Kim KJ, Yang I. Tumor response assessment by the single-lesion measurement per organ in small cell lung cancer. Chin J Cancer Res 2016;28(2):161-167. doi: 10.21147/j.issn.1000-9604.2016.02.03
25. Kus T, Aktas G, Sevinc A, et al. Prognostic impact of initial maximum standardized uptake value of (18)F-FDG $\mathrm{PET} / \mathrm{CT}$ on treatment response in patients with metastatic lung adenocarcinoma treated with erlotinib. Onco Targets Ther 2015;8:3749-56.

26. Schmitt RJ, Kreidler SM, Glueck DH, et al. Correlation between early 18F-FDG PET/CT response to BRAF and MEK inhibition and survival in patients with BRAFmutant metastatic melanoma. Nucl Med Commun 2016;37:122-8. 